\title{
Beauty Vector Meson Decay Constants from QCD Sum Rules
}

\author{
Wolfgang Lucha*, Dmitri Melikhov ${ }^{* \dagger}$ and Silvano Simula** \\ * Institute for High Energy Physics, Austrian Academy of Sciences, Nikolsdorfergasse 18, A-1050 Vienna, Austria \\ ${ }^{\dagger}$ D. V. Skobeltsyn Institute of Nuclear Physics, M. V. Lomonosov Moscow State University, 119991, Moscow, \\ Russia \\ ${ }^{* *}$ Istituto Nazionale di Fisica Nucleare, Sezione di Roma Tre, Via della Vasca Navale 84, I-00146, Roma, Italy
}

\begin{abstract}
We present the outcomes of a very recent investigation of the decay constants of nonstrange and strange heavy-light beauty vector mesons, with special emphasis on the ratio of any such decay constant to the decay constant of the corresponding pseudoscalar meson, by means of Borel-transformed QCD sum rules. Our results suggest that both these ratios are below unity.

Keywords: quantum chromodynamics, QCD sum rules, Borel sum rules, beauty mesons, heavy-meson decays, decay constants, pseudoscalar meson, vector meson, operator product expansion, Borel transformation, quark-hadron duality, renormalization scheme, renormalization scale PACS: $11.55 . \mathrm{Hx}, 12.38 . \mathrm{Lg}, 14.40 . \mathrm{Nd}, 03.65 . \mathrm{Ge}$
\end{abstract}

\section{INTRODUCTION: DECAY CONSTANTS OF VECTOR VS. PSEUDOSCALAR MESONS}

Recently, we started to apply the QCD sum-rule approach [1], modified by implementation of an improved algorithm of extracting hadron properties [2-5], to heavy-quark mesons. We analyzed the decay constants of the charmed and beauty pseudoscalar mesons $D, D_{s}, B, B_{s}[6,7]$, the bottom-quark mass value emerging from the beauty-meson decay constants $f_{B}, f_{B_{s}}$ [8], and the decay constants of the charmed vector mesons $D^{*}, D_{s}^{*}[9]$. The logically next step is the investigation of the decay constants $f_{B^{*}}, f_{B_{s}^{*}}$ of the vector beauty mesons $B^{*}, B_{s}^{*}[10]$. In this context, of preeminent interest is whether the decay constant of $B^{*}$ proves to be larger or smaller than that of $B$ (and the one of $B_{s}^{*}$ larger or smaller than that of $B_{s}$ ).

\section{RELATING HADRONIC FEATURES AND PARAMETERS OF UNDERLYING THEORY}

$Q C D$ sum rules are relations between observable properties of hadrons and the parameters of $\mathrm{QCD}$ - the quantum field theory governing strong interactions - , found by evaluating correlation functions of time-ordered nonlocal products of operators interpolating the hadron under consideration simultaneously at the hadronic level, by insertion of a complete set of states, and at the QCD level, by means of Wilson's operator product expansion (OPE). For a pseudoscalar beauty meson $B$ of mass $M_{B}$ composed of a bottom quark $b$ of mass $m_{b}$ and a light quark $q$ of mass $m_{q}$, let's interpolate $B$ by the pseudoscalar current $j_{5}(x)=\left(m_{b}+m_{q}\right) \bar{q}(x)$ i $\gamma_{5} b(x)$ defining the $B$-meson's decay constant $f_{B}$ by $\left\langle 0\left|j_{5}(0)\right| B\right\rangle=f_{B} M_{B}^{2}$ :

$$
\Pi\left(p^{2}\right) \equiv \mathrm{i} \int \mathrm{d}^{4} x \mathrm{e}^{\mathrm{i} p x}\left\langle 0\left|T\left(j_{5}(x) j_{5}^{\dagger}(0)\right)\right| 0\right\rangle .
$$

The resulting correlator $\Pi$ can be expressed in terms of a perturbative contribution of the form of a dispersion integral of a spectral density $\rho_{\text {pert }}$ and a non-perturbative (NP) or "power" contribution involving so-called "vacuum condensates". A Borel transformation to the Borel variable $\tau$ removes subtraction terms and suppresses higher hadronic contributions:

$$
\Pi(\tau)=f_{B}^{2} M_{B}^{4} \exp \left(-M_{B}^{2} \tau\right)+\int_{\left(M_{B^{*}}+M_{P}\right)^{2}}^{\infty} \mathrm{d} s \mathrm{e}^{-s \tau} \rho_{\text {hadr }}(s)=\int_{\left(m_{b}+m_{q}\right)^{2}}^{\infty} \mathrm{d} s \mathrm{e}^{-s \tau} \rho_{\text {pert }}(s, \mu)+\Pi_{\text {power }}(\tau, \mu) .
$$

The lower limit of the integral of the hadron spectral density $\rho_{\text {hadr }}$ involves the mass $M_{P}$ of the lightest relevant meson $P$. Assuming these higher hadronic contributions to compensate all the perturbative QCD contributions above the effective threshold $s_{\text {eff }}(\tau)$ results in the sum rule sought, where we take the liberty to label its QCD side the dual correlator $\Pi_{\text {dual }}$ :

$$
f_{B}^{2} M_{B}^{4} \exp \left(-M_{B}^{2} \tau\right)=\int_{\left(m_{b}+m_{q}\right)^{2}}^{s_{\text {eff }}(\tau)} \mathrm{d} s \mathrm{e}^{-s \tau} \rho_{\text {pert }}(s, \mu)+\Pi_{\text {power }}(\tau, \mu) \equiv \Pi_{\text {dual }}\left(\tau, s_{\text {eff }}(\tau)\right) .
$$


The perturbative-QCD spectral density, $\rho_{\text {pert }}$, in form of its expansion in powers of the strong coupling $\alpha_{\mathrm{s}}(\mu)$ in the $\overline{\mathrm{MS}}$ renormalization scheme involving the $b$-quark's pole mass $M_{b}$, is presently available up to three-loop accuracy [11,12]:

$$
\rho_{\text {pert }}(s, \mu)=\rho_{0}\left(s, M_{b}^{2}\right)+\frac{\alpha_{s}(\mu)}{\pi} \rho_{1}\left(s, M_{b}^{2}\right)+\frac{\alpha_{s}^{2}(\mu)}{\pi^{2}} \rho_{2}\left(s, M_{b}^{2}, \mu\right)+\cdots .
$$

Conversion to the $b$-quark's mass $\bar{m}_{b}(v)$ defined in the $\overline{\mathrm{MS}}$ renormalization scheme is effected, for known $r_{1}, r_{2}$ [13], by

$$
M_{b}=\bar{m}_{b}(v)\left(1+\frac{\alpha_{\mathrm{s}}(v)}{\pi} r_{1}+\frac{\alpha_{\mathrm{s}}^{2}(v)}{\pi^{2}} r_{2}+\cdots\right) .
$$

For generality, we allow the renormalization scales of the strong coupling, $\mu$, and of the $b$-quark mass, $v$, to be unequal.

Upon adopting as starting point of such QCD sum-rule analysis the interpolating vector current $j_{\mu}(x) \equiv \bar{q}(x) \gamma_{\mu} b(x)$, a (mutatis mutandis) similar formalism may be established for studying the features of the beauty vector mesons $B^{*}, B_{s}^{*}$.

\section{ADVANCED EXTRACTION OF HADRON FEATURES, SUCH AS DECAY CONSTANTS}

In the course of applying QCD sum rules, two steps demand particular consideration: the reasonable choice of the Borel variable and the determination of the behaviour of the effective continuum threshold as a function of this Borel variable.

Borel working window. The optimization of the extracted QCD sum-rule predictions for hadron observables provides two constraints that delimit, from both below and above, the interval of acceptable values of the Borel parameter $\tau$ : the relative contribution of the ground state (which increases with increasing value of $\tau$ ) to the correlator should be sufficiently large and the relative magnitude of the power corrections should remain under control (which becomes increasingly difficult for rising value of $\tau$ ). For, e.g., the pseudoscalar mesons $B$ and $B_{s}$, this confines $\tau$ to the range

$$
0.05 \mathrm{GeV}^{-2} \lesssim \tau \lesssim 0.175 \mathrm{GeV}^{-2}
$$

Effective continuum threshold. The effective threshold $s_{\text {eff }}(\tau)$ serves to encode the way all higher contributions from perturbative QCD are dealt with by assuming an exact cancellation against the hadronic excitations and continuum and thus constitutes, in addition to the perturbative spectral density and the power corrections, a crucial ingredient of the QCD sum-rule method. In order to formulate a criterion for finding the function $s_{\text {eff }}(\tau)$, we introduce, for the example of the $B$ meson, the ( $\tau$-dependent) dual mass $M_{\text {dual }}(\tau)$ and $d u a l$ decay constant $f_{\text {dual }}(\tau)$ by the definitions, obviously inspired by the particular way these two hadron characteristics enter in the Borelized QCD sum rule (1),

$$
M_{\text {dual }}^{2}(\tau) \equiv-\frac{\mathrm{d}}{\mathrm{d} \tau} \log \Pi_{\text {dual }}\left(\tau, s_{\text {eff }}(\tau)\right), \quad f_{\text {dual }}^{2}(\tau) \equiv \frac{\exp \left(M_{B}^{2} \tau\right)}{M_{B}^{4}} \Pi_{\text {dual }}\left(\tau, s_{\text {eff }}(\tau)\right)
$$

In order to infer the dependence on $\tau$ of the effective threshold from the knowledge of the meson mass $M_{B}$, we start from an ansatz for $s_{\text {eff }}(\tau)$, for which (because of the limited $\tau$ range) it is sufficient to use a polynomial of degree $n$,

$$
s_{\mathrm{eff}}^{(n)}(\tau)=\sum_{j=0}^{n} s_{j}^{(n)} \tau^{j}, \quad n=0,1,2, \ldots,
$$

and determine the expansion coefficients, $s_{j}^{(n)}$, by minimizing the squared differences of the squares of dual meson mass and measured meson mass over a set of $N$ equidistant points $\tau_{i}$ in the adopted Borel interval, i.e., the quantity

$$
\chi^{2} \equiv \frac{1}{N} \sum_{i=1}^{N}\left[M_{\text {dual }}^{2}\left(\tau_{i}\right)-M_{B}^{2}\right]^{2}, \quad N=1,2,3, \ldots
$$

\section{Uncertainties of decay constants from the advanced extraction algorithm for QCD sum rules}

All OPE parameters and the limited accuracy of the QCD sum-rule method add to the uncertainties of its predictions.

OPE-related error. Assuming Gaussian distributions for all parameters entering in the OPE except for the scales $\mu, v$, for which we assume uniform distributions in the range $3 \mathrm{GeV} \leq \mu, v \leq 6 \mathrm{GeV}$, bootstrapping yields a distribution of decay constants close to a Gaussian shape. Thus, we feel save if regarding the OPE-related error to be Gaussian.

Systematic error. We estimate this intrinsic error from the spread of results from linear through cubic ansatzes $s_{\text {eff }}^{(n)}(\tau)$. 


\section{DECAY CONSTANTS OF PSEUDOSCALAR $\left(f_{B}\right)$ VS. VECTOR BEAUTY MESONS $\left(f_{B^{*}}\right)$}

Having pinned down the variationally optimal effective-threshold polynomial $s_{\mathrm{eff}}^{(n)}(\tau)$ for fixed $n$, it is straightforward to extract the QCD sum-rule prediction for the dual decay constants of $B, B_{s}, B^{*}, B_{s}^{*}$. As OPE input, we use the more or less standard numerical values for $\overline{\mathrm{MS}}$-scheme quark masses, strong coupling, and lowest-dimensional vacuum condensates

$$
\begin{aligned}
& \bar{m}_{d}(2 \mathrm{GeV})=(3.5 \pm 0.5) \mathrm{MeV}, \quad \bar{m}_{s}(2 \mathrm{GeV})=(95 \pm 5) \mathrm{MeV}, \quad \bar{m}_{b}\left(\bar{m}_{b}\right)=(4.18 \pm 0.03) \mathrm{GeV}, \\
& \alpha_{\mathrm{s}}\left(M_{Z}\right)=0.1184 \pm 0.0007, \quad\langle\bar{q} q\rangle(2 \mathrm{GeV})=-[(269 \pm 17) \mathrm{MeV}]^{3}, \\
& \langle\bar{s} s\rangle(2 \mathrm{GeV})=(0.8 \pm 0.3)\langle\bar{q} q\rangle(2 \mathrm{GeV}), \quad\left\langle\frac{\alpha_{\mathrm{s}}}{\pi} G G\right\rangle=(0.024 \pm 0.012) \mathrm{GeV}^{4} .
\end{aligned}
$$

Optimization of our results requires careful choices of both renormalization scheme (defining the heavy-quark mass) and renormalization scale. In principle, QCD sum-rule results for observables should not depend on such technicalities. Truncations of perturbative expansions to finite powers of $\alpha_{\mathrm{s}}$ and of power corrections to vacuum condensates of lowest dimensions, however, induce a clearly unphysical dependence on renormalization details. Confidence in our findings is, of course, strengthened if we can arrange for a distinct hierarchy of the various contributions to the correlator. However, unlike the charmed-meson case, where we found only a mild renormalization-scale sensitivity, the beauty-meson decay constants and, in particular, their ratios $f_{B_{(s)}^{*}} / f_{B_{(s)}}$ are the result of a delicate [15] interplay of the different contributions.

\section{First point of reference: Dual decay constant $f_{B}$ of pseudoscalar nonstrange beauty meson $B$}

Figure 1, serving as solid basis of comparison, summarizes our $B$-meson decay-constant results arising for a constant effective threshold: relying on the $b$-quark $\overline{\mathrm{MS}}$ instead of pole mass and a sophisticated choice of $\mu$ is clearly preferable.

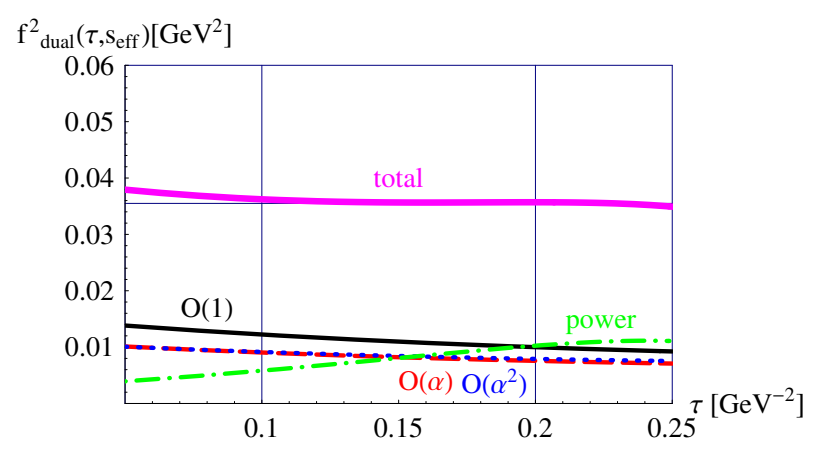

(a)

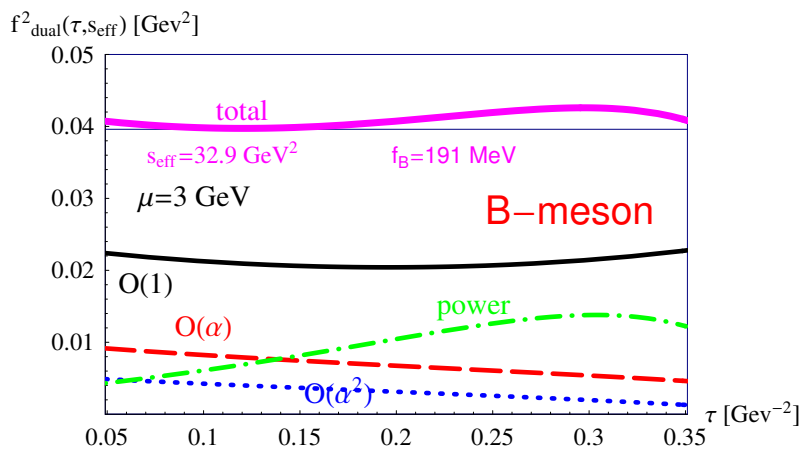

(c)

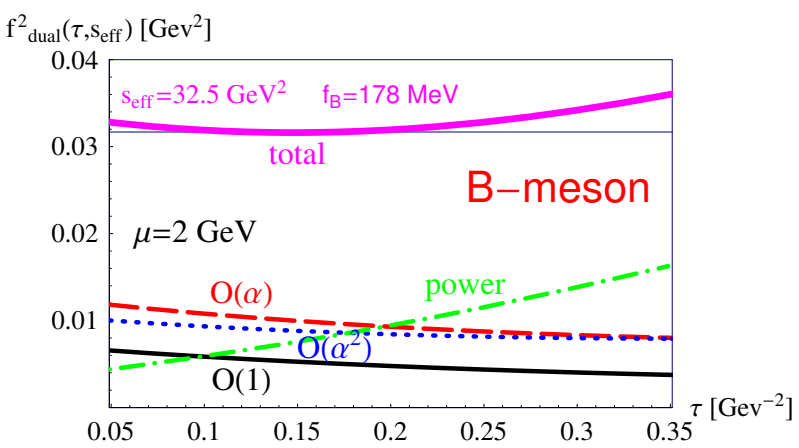

(b)

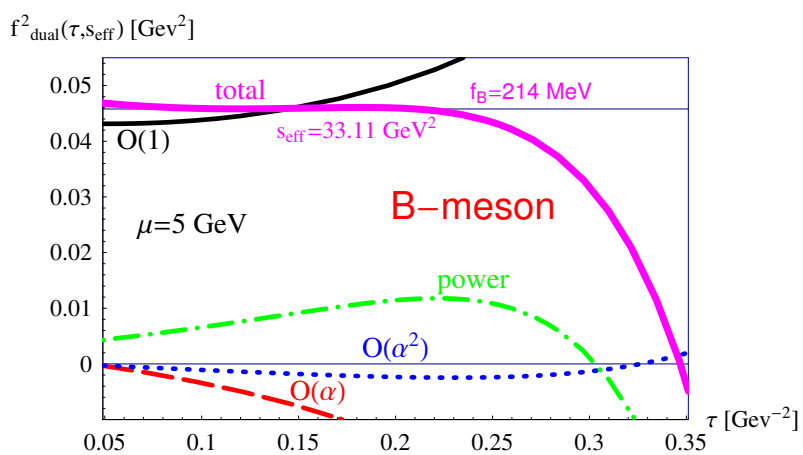

(d)

FIGURE 1. Dual decay constant $f_{\text {dual }}(\tau)$ of the $B$ meson: breakdown of the OPE contributions arising for fixed effective threshold $s_{\text {eff }}$ and either $b$-quark pole mass (a) or $b$-quark $\overline{\mathrm{MS}}$ mass at renormalization scales $\mu=2 \mathrm{GeV}(\mathrm{b}), \mu=3 \mathrm{GeV}$ (c), and $\mu=5 \mathrm{GeV}(\mathrm{d})$. 


\section{Actual primary target of interest: Decay constant $f_{B^{*}}$ of vector nonstrange beauty meson $B^{*}$}

Figure 2 illustrates the impact of definition of the heavy-quark mass and choice of renormalization scale on the decay constant of the beauty vector meson $B^{*}$ resulting for $s_{\text {eff }}(\tau)=$ const and the $b$-quark $\overline{\mathrm{MS}} \operatorname{mass} \bar{m}_{b}\left(\bar{m}_{b}\right)=4.18 \mathrm{GeV}[14]$ :

- Using the $b$-quark's pole mass, we obtain no perturbative hierarchy. All contributions are of roughly the same size.

- In the $\overline{\mathrm{MS}}$ scheme, perturbative convergence depends on $\mu$ and may be achieved by adopting a sufficiently large $\mu$.

- All plots shown in Fig. 2 exhibit, for the extracted decay constants, approximately flat plateaus over wide ranges of $\tau$. This observation tells us (anew) that the requirement of Borel stability does not necessarily yield reliable results.

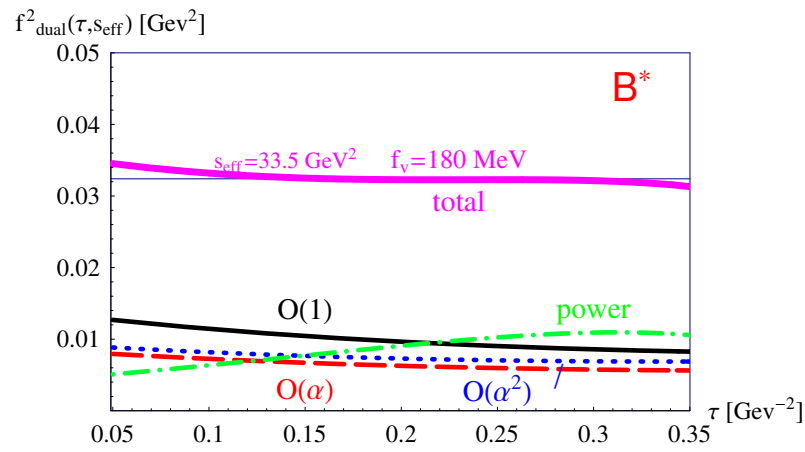

(a)

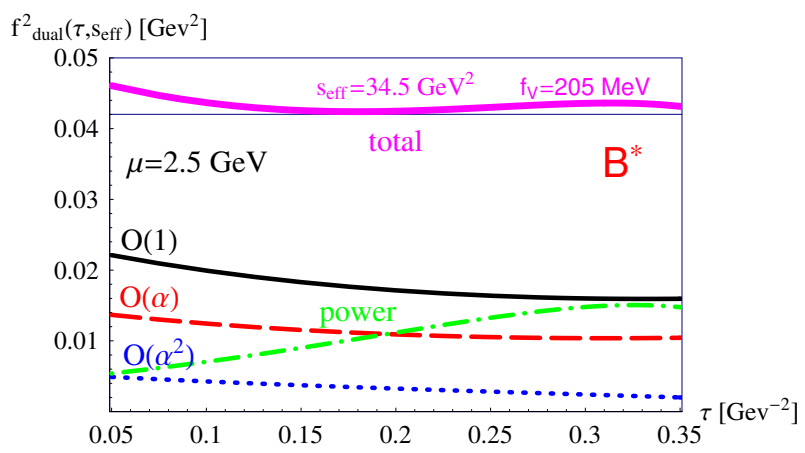

(c)

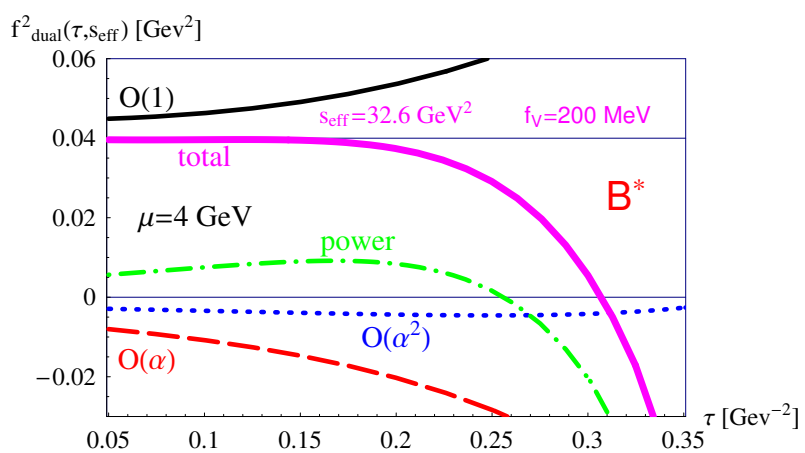

(e)

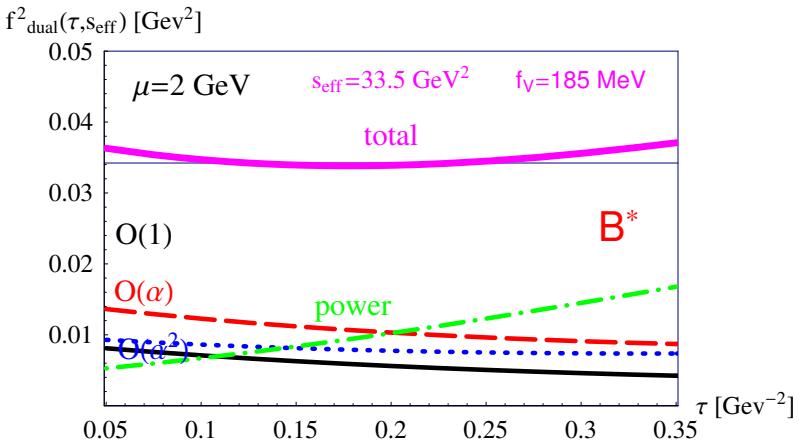

(b)

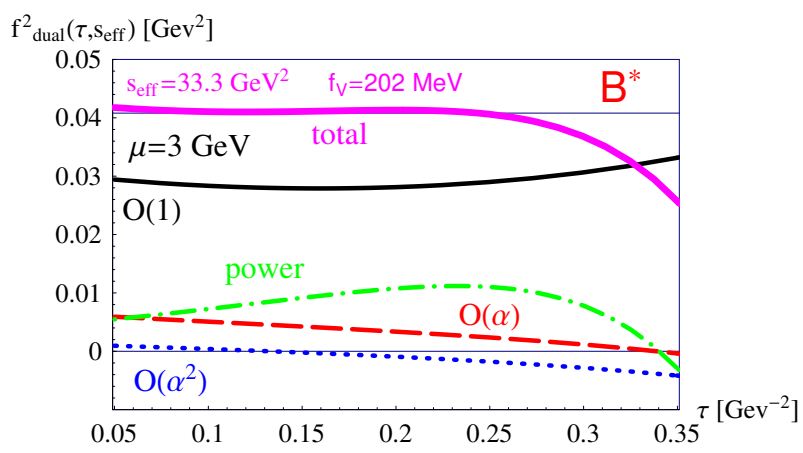

(d)

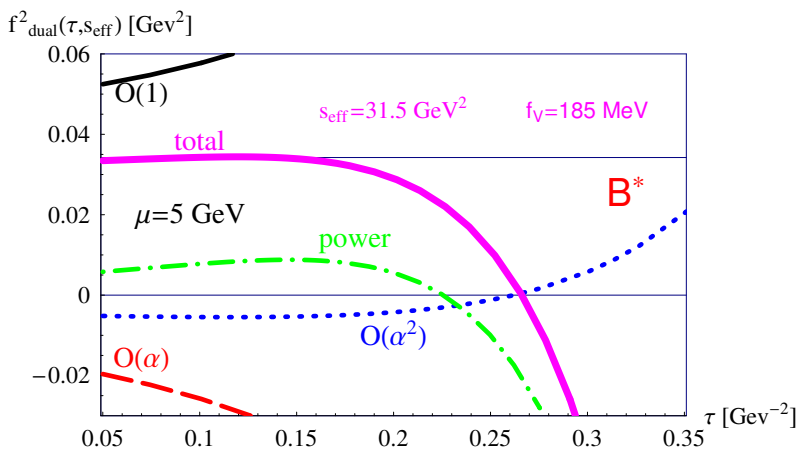

(f)

FIGURE 2. Dual decay constant $f_{\text {dual }}(\tau)$ of the $B^{*}$ meson: breakdown of the OPE contributions, found if adopting a fixed effective threshold $s_{\text {eff }}$ and either the pole-mass (a) or the $\overline{\mathrm{MS}}$-mass definition of the $b$-quark's mass, for several choices of the renormalization scale in the interval $2 \mathrm{GeV} \leq \mu \leq 5 \mathrm{GeV}$, namely, $\mu=2 \mathrm{GeV}$ (b), $\mu=2.5 \mathrm{GeV}$ (c), $\mu=3 \mathrm{GeV}$ (d), $\mu=4 \mathrm{GeV}$ (e) and $\mu=5 \mathrm{GeV}$ (f). 


\section{DEPENDENCE OF THE DECAY CONSTANTS ON THE RENORMALIZATION SCALE}

The strong sensitivity of $f_{B}$ to the $b$-quark mass allowed us to deduce the latter [8] from the lattice-QCD findings for $f_{B}$ :

$$
\bar{m}_{b}\left(\bar{m}_{b}\right)=\left(4.247 \pm 0.027_{\mathrm{OPE}} \pm 0.018_{\mathrm{exp}} \pm 0.011_{\mathrm{syst}}\right) \mathrm{GeV}=(4.247 \pm 0.034) \mathrm{GeV} \text {. }
$$

The behaviour of our numerical results for the dual decay constants of the nonstrange beauty mesons as functions of the renormalization scale $\mu$ in the proximity of an average $\bar{\mu}$ of $\mu$ defined by requiring $f_{\text {dual }}(\bar{\mu})=\left\langle f_{\text {dual }}(\mu)\right\rangle$ (cf. Fig. 3) and as functions of the $\overline{\mathrm{MS}} b$-quark mass $m_{b} \equiv \bar{m}_{b}\left(\bar{m}_{b}\right)$ may be approximately reproduced by a linear decrease with $m_{b}$ and a series expansion in powers of the logarithm of $\mu$. For the pseudoscalar meson $B$, the $\mu$ dependence is not very dramatic:

$$
\begin{aligned}
& f_{B}^{\text {dual }}\left(m_{b}, \mu=\bar{\mu}\right)=192.6 \mathrm{MeV}-13 \mathrm{MeV}\left(\frac{m_{b}-4.247 \mathrm{GeV}}{0.034 \mathrm{GeV}}\right), \quad \bar{\mu}=5.59 \mathrm{GeV}, \\
& f_{B}^{\text {dual }}\left(m_{b}=4.247 \mathrm{GeV}, \mu\right)=192.6 \mathrm{MeV}\left(1-0.0015 \log \frac{\mu}{\bar{\mu}}+0.030 \log ^{2} \frac{\mu}{\bar{\mu}}+0.061 \log ^{3} \frac{\mu}{\bar{\mu}}\right) .
\end{aligned}
$$

For the vector meson $B^{*}$, the coefficients are markedly larger, that of the linear term by almost two orders of magnitude:

$$
\begin{aligned}
& f_{B^{*}}^{\text {dual }}\left(m_{b}, \mu=\bar{\mu}\right)=186.4 \mathrm{MeV}-10 \mathrm{MeV}\left(\frac{m_{b}-4.247 \mathrm{GeV}}{0.034 \mathrm{GeV}}\right), \quad \bar{\mu}=5.82 \mathrm{GeV}, \\
& f_{B^{*}}^{\text {dual }}\left(m_{b}=4.247 \mathrm{GeV}, \mu\right)=186.4 \mathrm{MeV}\left(1+0.106 \log _{\frac{\mu}{\bar{\mu}}}+0.337 \log ^{2} \frac{\mu}{\bar{\mu}}+0.173 \log ^{3} \frac{\mu}{\bar{\mu}}\right) .
\end{aligned}
$$

The very pronounced dependence of the $B^{*}$ decay constant on the renormalization scale $\mu$ has, of course, a considerable impact on the ratio $f_{B^{*}} / f_{B}$ of beauty-meson vector-to-pseudoscalar decay constants: this ratio proves to emerge slightly above unity for small $\mu$ (say, $\mu \lesssim 3 \mathrm{GeV}$ ) but certainly below unity for larger $\mu$ (i.e., $\mu \gtrsim 3 \mathrm{GeV}$ ), as evident from Fig. 3 .

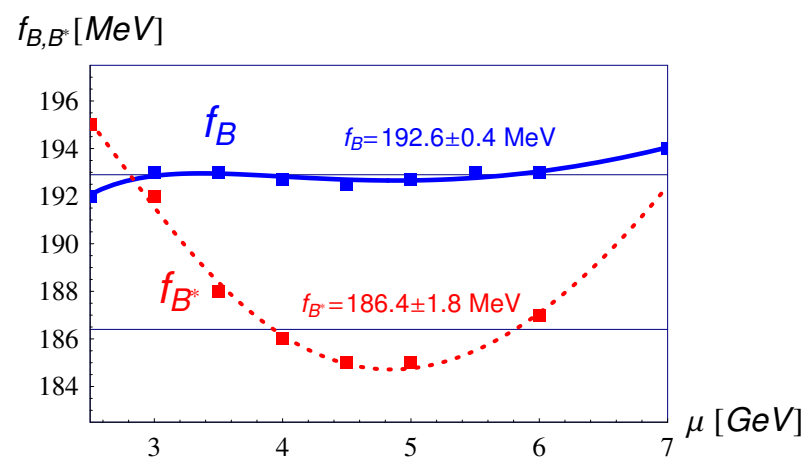

(a)

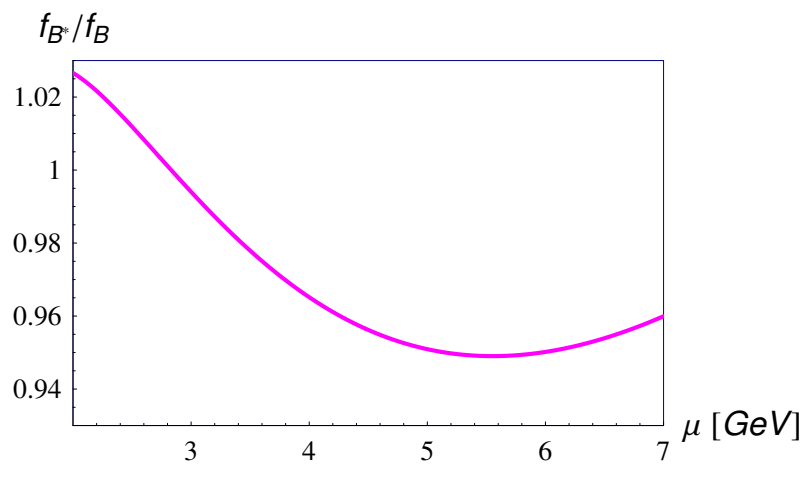

(b)

FIGURE 3. Dependence of our results for the decay constants $f_{B}, f_{B^{*}}\left(\right.$ a) and their ratio $f_{B^{*}} / f_{B}$ (b) on the renormalization scale $\mu$.

\section{CONDENSED SUMMARY OF OUTCOMES, DISCUSSION, CONCLUSION, OUTLOOK}

The extraction of the decay constants of heavy-light mesons from Borelized QCD sum rules forms in the beauty-meson sector, for, at least, two reasons, a definitely greater challenge than it does in the charmed-meson sector. First, for beauty mesons, we encounter a pronounced dependence of our predictions on the mass of the $b$ quark. Secondly, for the beauty mesons, the predictions develop an unpleasantly strong dependence on the adopted renormalization scale $\mu$. Inspecting the behaviour of the decay constants under variation of $\mu$, we find that the decay constant of the pseudoscalar meson $B$, $f_{B}$, increases with rising $\mu$ (see Fig. 1), whereas the one of the vector meson $B^{*}, f_{B^{*}}$, decreases with rising $\mu$ (cf. Fig. 2). Averaging our findings for a quadratic effective-threshold polynomial (2) over the interval $3 \mathrm{GeV} \leq \mu \leq 6 \mathrm{GeV}$, we find

$$
\begin{array}{rlrl}
f_{B} & =(192.6 \pm 1.6) \mathrm{MeV}, & & f_{B^{*}}=(186.4 \pm 3.2) \mathrm{MeV} \\
f_{B_{s}} & =(231.0 \pm 1.8) \mathrm{MeV}, & f_{B_{s}^{*}}=(215.2 \pm 3.0) \mathrm{MeV}
\end{array}
$$


where the errors take into account only the effects of the variation of $\mu$. The resulting decay-constant ratios (Fig. 3) read

$$
\frac{f_{B^{*}}}{f_{B}}=0.923 \pm 0.059, \quad \frac{f_{B_{s}^{*}}}{f_{B_{s}}}=0.932 \pm 0.047 .
$$

Surprisingly, the QCD sum-rule prediction for $f_{B^{*}} / f_{B}$ is below the corresponding results from lattice QCD, which seem to favour a value slightly above but still close to unity [16]. Clearly, such tension calls for further detailed investigations.

\section{REFERENCES}

1. M. A. Shifman, A. I. Vainshtein, and V. I. Zakharov, Nucl. Phys. B 147 (1979) 385.

2. W. Lucha, D. Melikhov, and S. Simula, Phys. Rev. D 79 (2009) 096011, arXiv:0902.4202 [hep-ph].

3. W. Lucha, D. Melikhov, and S. Simula, J. Phys. G 37 (2010) 035003, arXiv:0905.0963 [hep-ph].

4. W. Lucha, D. Melikhov, and S. Simula, Phys. Lett. B 687 (2010) 48, arXiv: 0912.5017 [hep-ph].

5. W. Lucha, D. I. Melikhov, and S. Simula, Phys. Atom. Nucl. 73 (2010) 1770, arXiv:1003.1463 [hep-ph].

6. W. Lucha, D. Melikhov, and S. Simula, J. Phys. G 38 (2011) 105002, arXiv:1008.2698 [hep-ph].

7. W. Lucha, D. Melikhov, and S. Simula, Phys. Lett. B 701 (2011) 82, arXiv:1101.5986 [hep-ph].

8. W. Lucha, D. Melikhov, and S. Simula, Phys. Rev. D 88 (2013) 056011, arXiv:1305.7099 [hep-ph].

9. W. Lucha, D. Melikhov, and S. Simula, Phys. Lett. B 735 (2014) 12, arXiv:1404.0293 [hep-ph].

10. W. Lucha, D. Melikhov, and S. Simula, Vienna preprint HEPHY-PUB 944/14 (2014), in preparation.

11. K. G. Chetyrkin and M. Steinhauser, Phys. Lett. B 502 (2001) 104, arXiv:hep-ph/0012002.

12. K. G. Chetyrkin and M. Steinhauser, Eur. Phys. J. C 21 (2001) 319, arXiv:hep-ph/0108017.

13. M. Jamin and B. O. Lange, Phys. Rev. D 65 (2002) 056005, arXiv:hep-ph/0108135.

14. K. A. Olive et al. (Particle Data Group), Chin. Phys. C 38 (2014) 090001.

15. W. Lucha, D. Melikhov, and S. Simula, EPJ Web of Conferences 80 (2014) 00046, arXiv:1410.6684 [hep-ph].

16. W. Lucha, D. Melikhov, and S. Simula, arXiv:1411.3890 [hep-ph]. 